\title{
Radiation from an oscillator uniformly moving along the axis of a dielectric cylinder
}

\author{
A. A. Saharian* \\ Department of Physics, Yerevan State University, 375049 Yerevan, Armenia \\ and Institute of Applied Problems in Physics, 375014 Yerevan, Armenia \\ and \\ A. S. Kotanjyan \\ Institute of Applied Problems in Physics, 375014 Yerevan, Armenia
}

November 9, 2018

\begin{abstract}
The radiation generated by a charged longitudinal oscillator moving with a constant drift velocity along the axis of a dielectric cylinder immersed in a homogeneous medium is investigated. For an arbitrary oscillation law a formula is derived for the spectral-angular distribution of this radiation. Under the Cherenkov condition for the dielectric permittivity of the external medium and oscillator drift velocity this formula contains two summands. The first one corresponds to the radiation with a continuous spectrum which propagates at the Cherenkov angle of the external medium. The second one describes the radiation which has a discrete spectrum for a given angle of propagation. The corresponding frequencies are multiples of the Doppler-shifted oscillation frequency. The results of numerical calculations for the angular distribution of the radiated quanta are presented and they are compared with the corresponding quantities for the radiation in a homogeneous medium. It is shown that the presence of the cylinder can increase essentially the radiation intensity.
\end{abstract}

PACS number(s): 41.60.-m, 41.60.Bq

\section{Introduction}

The operation of a number of devices assigned to production of electromagnetic radiation is based on the radiation from periodically moving charged particles, in particular, longitudinal and transversal oscillators. Well-known examples are undulators, wigglers and free-electron lasers (see, for instance, Refs. [1, 2, 3, 4]). The extensive applications of such devices motivate the importance of investigations for various mechanisms of controlling the radiation parameters. From this point of view, it is of interest to investigate the influence of medium on the spectral and angular distributions of the radiation. The presence of matter alters the radiation field and can give rise to new types of radiation processes. This study is also important with respect to some astrophysical problems.

Interfaces of media are widely used to control the radiation flow emitted by various systems. Well-known examples of such a kind are the Cherenkov radiation of a charge moving parallel to a plane interface of two media or flying parallel to the axis of a dielectric cylinder, transition

*Email address: saharyan@server.physdep.r.am 
radiation, Smith-Purcell radiation. In Refs. [5] the radiation from a charged particle flying over a surface acoustic wave generated on a plane interface between two media is investigated. The corresponding radiation from electron bunches and coherence effects are considered in Ref. [6]. In a series of papers started in Refs. $[7,8]$ we have considered the most simple geometries of boundaries, namely, the boundaries with spherical and cylindrical symmetries. The radiation from a charge rotating around a dielectric ball enclosed by a homogeneous medium is investigated in Refs. $[8,9]$. It has been shown that the interference between the synchrotron and Cherenkov radiations leads to interesting effects: if for the material of the ball and particle velocity the Cherenkov condition is satisfied, there are strong narrow peaks in the radiation intensity. At these peaks the radiated energy exceeds the corresponding quantity in the case of a homogeneous medium by some orders of magnitude. A similar problem for the case of the cylindrical symmetry has been considered in Refs. [7, 10, 11, 12, 13]. In Ref. [7] a recurrent scheme is developed for constructing the Green function of the electromagnetic field for a medium consisting of an arbitrary number of coaxial cylindrical layers. The investigation of the radiation from a charged particle circulating around or inside a dielectric cylinder immersed in a homogeneous medium, has shown that under the Cherenkov condition for the material of the cylinder and the velocity of the particle, there are narrow peaks in the angular distribution of the number of quanta emitted into the exterior space. For some values of the parameters the density of the number of quanta in these peaks exceeds the corresponding quantity for the radiation in vacuum by several orders.

In the present paper, on the basis of the Green function obtained in Ref. [7], the radiation by a longitudinal oscillator with an arbitrary law of oscillations uniformly moving along the axis of a dielectric cylinder is studied. The radiation of charged oscillators in vacuum and inhomogeneous media was considered in a number of papers (see, e.g., [4], [14]-[18], and references therein) in connection with many possible applications (generation of radiation, detection of high-energy particles, etc.). In particular, the radiation from an oscillator with harmonic oscillations in the laboratory frame in an infinite periodic medium was studied in Refs. [14, 15]. The spectralangular distribution of the radiation intensity of an oscillator moving over interface of two media was considered in Ref. [16]. The radiation by a longitudinal oscillator uniformly moving along the axis of a cylindrical waveguide is investigated in Refs. $[18,19]$. The radiation properties in vacuum for the most general case of harmonic oscillations in a four dimensional form are described in Ref. [4]. The special case of a longitudinal oscillator with harmonic oscillations in the proper reference frame is considered in Ref. [17].

This paper is organized as follows. In next section the expressions for the vector-potential and electromagnetic fields are derived for an arbitrary law of oscillation by using the Green function. The angular-frequency distribution for the corresponding radiation intensity is investigated in Sec. 3. Two examples for the oscillatory motion corresponding to harmonic oscillations in the laboratory and proper reference frames are considered in Sec. 4. Section 5 concludes the main results of the paper.

\section{Oscillator fields}

Let a point charge $q$ moves along the axis of a dielectric cylinder with permittivity $\varepsilon_{0}$ and radius $\rho_{1}$. This system is immersed in a homogeneous medium with dielectric permittivity $\varepsilon_{1}$. We consider the case when the charged particle executes longitudinal oscillations with a drift of the constant velocity $v_{0}$ :

$$
z=v_{0} t+f(t), \quad x=y=0,
$$

where the Cartesian axis $z$ coincides with the cylinder axis, and $f(t)$ is an arbitrary periodic function with the period $T=2 \pi / \omega_{0}$. In a properly chosen cylindrical coordinate system $(\rho, \phi, z)$ 
the spatial part of the four-vector of the current density created by this charge is written as

$$
j_{l}=\frac{q}{\rho} v_{0} \delta(\rho) \delta(\phi) \delta\left(z-v_{0} t-f(t)\right) \delta_{l z,} \quad l=\rho, \phi, z .
$$

The corresponding solution of the Maxwell equations for the four-vector potential is expressed in terms of the Green function $G_{i l}\left(\mathbf{r}, t, \mathbf{r}^{\prime}, t^{\prime}\right)$ of the electromagnetic field, which is a second-rank tensor:

$$
A_{i}(\mathbf{r}, t)=-\frac{1}{2 \pi^{2} c} \int G_{i l}\left(\mathbf{r}, t, \mathbf{r}^{\prime}, t^{\prime}\right) j_{l}\left(\mathbf{r}^{\prime}, t\right) d \mathbf{r}^{\prime} d t^{\prime}, \quad l=t, \rho, \phi, z .
$$

Taking into account the cylindrical symmetry of the problem under consideration, it is convenient to present the Green function as a Fourier expansion

$$
G_{i l}\left(\mathbf{r}, t, \mathbf{r}^{\prime} t^{\prime}\right)=\sum_{m=-\infty}^{\infty} \int_{-\infty}^{\infty} d k_{z} d \omega G_{i l}\left(m, k_{z}, \omega, \rho, \rho^{\prime}\right) \exp \left[i m\left(\phi-\phi^{\prime}\right)+i k_{z}\left(z-z^{\prime}\right)-i \omega\left(t-t^{\prime}\right)\right]
$$

For a dielectric cylinder with permittivity $\varepsilon_{0}$ immersed in a homogeneous medium with permittivity $\varepsilon_{1}$, the Fourier transform of the Green function entering in Eq. (4) is given in Ref. [7]. When $\rho^{\prime}<\rho_{1}$, the function $G_{i l}\left(m, k_{z}, \omega, \rho, \rho^{\prime}\right)$ depends on $\rho^{\prime}$ via the Bessel function $J_{m}\left(\lambda_{0} \rho^{\prime}\right)$. For brevity, here and below we use the notation

$$
\lambda_{j}^{2}=\frac{\omega^{2}}{c^{2}} \varepsilon_{j}-k_{z}^{2}, \quad j=0,1
$$

Due to the presence of the function $\delta\left(\rho^{\prime}\right)$ in expression (2) for the current density, only the term with $m=0$ gives the nonzero contribution to the vector-potential in Eq. (3). As a result, the field does not depend on the angle $\phi$. This is a simple consequence of the azimuthal symmetry for the problem under consideration. Thus, in this paper it is sufficient to have expressions for $G_{i z}\left(m=0, k_{z}, \omega, \rho, \rho^{\prime}=0\right) \equiv G_{i z}\left(k_{z}, \omega, \rho\right)$. In the Lorentz gauge these expressions are immediately derived from the general formulas of Ref. [7] and have the form

$$
\begin{aligned}
G_{\rho z}\left(k_{z}, \omega, \rho\right) & =-i \frac{\left(1-\varepsilon_{0} / \varepsilon_{1}\right) k_{z} H_{0}\left(\lambda_{1} \rho_{1}\right)}{\rho_{1} \lambda_{0} W_{\varepsilon}\left(J_{0}, H_{0}\right) W\left(J_{0}, H_{0}\right)} J_{1}\left(\lambda_{0} \rho_{<}\right) H_{1}\left(\lambda_{1} \rho_{>}\right), \rho_{>}=\min _{\max }\left(\rho, \rho_{1}\right), \\
G_{\phi z}\left(k_{z}, \omega, \rho\right) & =0, \\
G_{z z}\left(k_{z}, \omega, \rho\right) & =\frac{\pi}{2 i W\left(J_{0}, H_{0}\right)} \begin{cases}W\left(J_{0}, H_{0}\right) H_{0}\left(\lambda_{0} \rho\right)-W\left(H_{0}, H_{0}\right) J_{0}\left(\lambda_{0} \rho\right), & \rho<\rho_{1}, \\
2 i H_{0}\left(\lambda_{1} \rho\right) / \pi \rho_{1}, & \rho>\rho_{1},\end{cases}
\end{aligned}
$$

where $H_{m}(x)=H_{m}^{(1)}(x)$ is the Hankel function of the first kind. In these formulas the following notations are used:

$$
\begin{aligned}
W(a, b) & =a\left(\lambda_{0} \rho_{1}\right) \frac{\partial b\left(\lambda_{1} \rho_{1}\right)}{\partial \rho_{1}}-b\left(\lambda_{1} \rho_{1}\right) \frac{\partial a\left(\lambda_{0} \rho_{1}\right)}{\partial \rho_{1}} \\
W_{\varepsilon}\left(J_{0}, H_{0}\right) & =J_{0}\left(\lambda_{0} \rho_{1}\right) H_{1}\left(\lambda_{1} \rho_{1}\right)-\frac{\varepsilon_{0} \lambda_{1}}{\varepsilon_{1} \lambda_{0}} J_{1}\left(\lambda_{0} \rho_{1}\right) H_{0}\left(\lambda_{1} \rho_{1}\right) .
\end{aligned}
$$

In the definition of $\lambda_{1}$ from Eq. (5) one should take into account that in the presence of the imaginary part $\varepsilon_{1}^{\prime \prime}(\omega)$ for the dielectric permittivity $\left(\varepsilon_{1}=\varepsilon_{1}^{\prime}+i \varepsilon_{1}^{\prime \prime}\right)$ the radiation field in the exterior medium must damp exponentially for large $\rho$. This leads to the following relations:

$$
\lambda_{1}=\left\{\begin{array}{c}
(\omega / c) \sqrt{\varepsilon_{1}-k_{z}^{2} c^{2} / \omega^{2}}, \quad \omega^{2} \varepsilon_{1} / c^{2}>k_{z}^{2}, \\
i \sqrt{k_{z}^{2}-\omega^{2} \varepsilon_{1} / c^{2}}, \quad \omega^{2} \varepsilon_{1} / c^{2}<k_{z}^{2} .
\end{array}\right.
$$

Note that for $\lambda_{1}^{2}>0$ the sign of $\lambda_{1}$ may be also determined from the principle of radiation (different signs of $\omega t$ and $\lambda_{1} \rho$ in expressions for the fields) for large $\rho$. 
Substituting Eq. (2) into formula (3), the following expressions are obtained for the components of the vector-potential:

$$
A_{l}(\rho, z, t)=-\frac{q}{\pi c} \sum_{n=-\infty}^{\infty} \int_{-\infty}^{\infty} d k_{z} \exp \left[i\left(z k_{z}-\omega_{n}\left(k_{z}\right) t\right)\right] G_{l z}\left(k_{z}, \omega_{n}\left(k_{z}\right), \rho\right) g_{n}\left(k_{z}\right) \frac{\omega_{n}\left(k_{z}\right)}{k_{z}}
$$

where

$$
\omega_{n}\left(k_{z}\right)=n \omega_{0}+k_{z} v_{0},
$$

and $g_{n}\left(k_{z}\right)$ is the Fourier transform of the function $e^{i k_{z} f(t)}$ :

$$
e^{i k_{z} f(t)}=\sum_{n=-\infty}^{+\infty} g_{n}\left(k_{z}\right) e^{i n \omega_{0} t}, \quad g_{n}\left(k_{z}\right)=\frac{1}{T} \int_{-T / 2}^{T / 2} e^{i k_{z} f(t)-i n \omega_{0} t} d t
$$

From the reality of the function $f(t)$ it follows that $g_{-n}\left(-k_{z}\right)=g_{n}^{*}\left(k_{z}\right)$. Fields inside and outside the waveguide are derived by making use of the corresponding expressions (6) for the components of the reduced Green function. Below we will consider the fields in the region $\rho>\rho_{1}$. Taking into account expressions (6) and formula (9), for the vector-potential in this region one obtains

$$
A_{l}(\rho, z, t)=\sum_{n=-\infty}^{+\infty} \int_{-\infty}^{\infty} d k_{z} \exp \left[i\left(k_{z} z-\omega_{n}\left(k_{z}\right) t\right)\right] A_{n l}\left(k_{z}, \rho\right), \quad l=\rho, z, \phi,
$$

where the coefficients $A_{n l}$ are determined by the relations

$$
\begin{aligned}
& A_{n \rho}=\frac{i q\left(1-\varepsilon_{0} / \varepsilon_{1}\right)}{\pi c \rho_{1}} \frac{\omega_{n}\left(k_{z}\right) H_{0}\left(\lambda_{1} \rho_{1}\right) J_{1}\left(\lambda_{0} \rho_{1}\right)}{\lambda_{0} W_{\varepsilon}\left(J_{0}, H_{0}\right) W\left(J_{0}, H_{0}\right)} g_{n}\left(k_{z}\right) H_{1}\left(\lambda_{1} \rho\right), \\
& A_{n z}=-\frac{q}{\pi c \rho_{1}} \frac{\omega_{n}\left(k_{z}\right) g_{n}\left(k_{z}\right)}{k_{z} W\left(J_{0}, H_{0}\right)} H_{0}\left(\lambda_{1} \rho\right), \\
& A_{n \phi}=0,
\end{aligned}
$$

and in the definitions of $\lambda_{j}$ one has to substitute $\omega=\omega_{n}\left(k_{z}\right)$. From the Lorentz gauge condition, by using the symmetry of the problem under consideration, we obtain for the scalar potential:

$$
\varphi_{n}\left(k_{z}, \rho\right)=\left[\frac{1}{\rho} \frac{\partial\left(\rho A_{n \rho}\right)}{\partial \rho}+i k_{z} A_{n z}\right] \frac{c}{i \omega_{n}\left(k_{z}\right) \varepsilon_{1}} .
$$

As is seen from formula (12), analogous expressions may also be written for the electric and magnetic fields. Having expressions for the Fourier coefficients of the vector- and scalar-potentials, one can derive the corresponding expressions for these fields:

$$
\begin{aligned}
& E_{n \rho}=\frac{q g_{n}\left(k_{z}\right)}{\pi \rho_{1} \varepsilon_{1} W_{\varepsilon}\left(J_{0}, H_{0}\right)} H_{1}\left(\lambda_{1} \rho\right), \quad E_{n z}=\frac{i q \lambda_{1} g_{n}\left(k_{z}\right)}{\pi \rho_{1} \varepsilon_{1} k_{z} W_{\varepsilon}\left(J_{0}, H_{0}\right)} H_{0}\left(\lambda_{1} \rho\right), \quad E_{n \phi}=0, \\
& H_{n \phi}=\frac{q \omega_{n}\left(k_{z}\right) g_{n}\left(k_{z}\right)}{\pi c \rho_{1} k_{z} W_{\varepsilon}\left(J_{0}, H_{0}\right)} H_{1}\left(\lambda_{1} \rho\right), \quad H_{n \rho}=H_{n z}=0 .
\end{aligned}
$$

As follows from these formulas, $\mathbf{E}_{n} \cdot \mathbf{H}_{n}=0$, i.e., the Fourier components of the electric and magnetic fields are perpendicular, and one can write

$$
\mathbf{E}_{n}=\left[\mathbf{b} \mathbf{H}_{n}\right], \quad \mathbf{b}=-\frac{c}{\omega_{n}\left(k_{z}\right) \varepsilon_{1}}\left(\lambda_{1}, 0, k_{z}\right) .
$$

As functions of $k_{z}$ the Fourier coefficients for the fields determined by relations (15), (16) have poles corresponding to the zeros of the function $W_{\varepsilon}\left(J_{0}, H_{0}\right)$. It can be seen that this function has zeros only for $\lambda_{1}^{2}<0<\lambda_{0}^{2}$. As a necessary condition for this one has $\varepsilon_{1}<\varepsilon_{0}$. Note that for the corresponding modes the coefficients (15), (16) are proportional to the MacDonald function $K_{m}\left(\left|\lambda_{1}\right| \rho\right), m=0,1$, and they are exponentially damped with the distance from the cylinder axis. These modes are precisely the eigenmodes of the dielectric cylinder and propagate inside the dielectric cylinder. Below, in the consideration of the intensity for the radiation to the exterior medium, we will disregard the contribution of the poles corresponding to these modes. 


\section{Spectral-angular distribution of the radiation intensity}

The average energy flux per unit time through the cylindrical surface of radius $\rho$ coaxial with the dielectric cylinder is given by the Poynting vector $\mathbf{S}$ as

$$
I=\frac{2 \pi}{T} \int_{0}^{T} d t \int_{-\infty}^{\infty}\left(\mathbf{S n}_{\rho}\right) \rho d z, \quad \mathbf{S}=\frac{c}{4 \pi}[\mathbf{E H}], \quad T=\frac{2 \pi}{\omega_{0}},
$$

where $\mathbf{n}_{\rho}$ is the unit vector of the cylindrical surface. By making use of formulas (12), (15), and (16), we obtain

$$
I=-\frac{i q^{2} \rho}{\pi \rho_{1}^{2}} \sum_{n=-\infty}^{+\infty} \int_{-\infty}^{\infty} d k_{z} \frac{\lambda_{1} \omega_{n}\left(k_{z}\right)\left|g_{n}\left(k_{z}\right)\right|^{2}}{\varepsilon_{1} k_{z}^{2}\left|W_{\varepsilon}\left(J_{0}, H_{0}\right)\right|^{2}} H_{0}\left(\lambda_{1} \rho\right) H_{1}^{*}\left(\lambda_{1}^{*} \rho\right) .
$$

Using the fact that the replacement $n \rightarrow-n, k_{z} \rightarrow-k_{z}$ leads to $\omega_{n}\left(k_{z}\right) \rightarrow-\omega_{n}\left(k_{z}\right), \lambda_{1} \rightarrow-\lambda_{1}^{*}$ and $\left|g_{-n}\left(-k_{z}\right)\right|^{2}=\left|g_{n}\left(k_{z}\right)\right|^{2}$, expression (19) can be written in the explicitly real form

$$
I=\frac{2 q^{2} \rho}{\pi \rho_{1}^{2}} \sum_{n=0}^{\infty} \int_{-\infty}^{\infty} d k_{z} \frac{\omega_{n}\left(k_{z}\right)\left|g_{n}\left(k_{z}\right)\right|^{2}}{\varepsilon_{1} k_{z}^{2}\left|W_{\varepsilon}\left(J_{0}, H_{0}\right)\right|^{2}} \operatorname{Im}\left[\lambda_{1} H_{0}\left(\lambda_{1} \rho\right) H_{1}^{*}\left(\lambda_{1}^{*} \rho\right)\right],
$$

where the prime over the sum means that the term with $n=0$ must be taken with the weight 1/2. Now by using Eq.(8) and the Wronskian for the Bessel functions, it can be easily seen that

$$
\operatorname{Im}\left[\lambda_{1} H_{0}\left(\lambda_{1} \rho\right) H_{1}^{*}\left(\lambda_{1}^{*} \rho\right)= \begin{cases}2 / \pi \rho, & \lambda_{1}^{2}>0 \\ 0, & \lambda_{1}^{2}<0 .\end{cases}\right.
$$

Hence, for the energy flux one obtains

$$
I=\frac{4 q^{2}}{\pi^{2} \rho_{1}^{2}} \sum_{n=0}^{\infty} \int_{\lambda_{1}^{2}>0} d k_{z} \frac{\omega_{n}\left(k_{z}\right)\left|g_{n}\left(k_{z}\right)\right|^{2}}{\varepsilon_{1} k_{z}^{2}\left|W_{\varepsilon}\left(J_{0}, H_{0}\right)\right|^{2}} .
$$

As we could expect in the absence of absorption this flux does not depend on the distance $\rho$ from the cylinder axis.

We first discuss the case $\omega_{0}=0$, which corresponds to the uniform motion of the charge along the axis of a dielectric cylinder. Then, according to Eqs. (5) and (10), $\omega_{n}\left(k_{z}\right)$ and $\lambda_{j}$ do not depend on $n$, and the series in Eq. (20) is easily summarized by the standard formula for the Fourier transformation:

$$
\sum_{n=-\infty}^{+\infty}\left|g_{n}\left(k_{z}\right)\right|^{2}=\frac{1}{T} \int_{-T / 2}^{T / 2}\left|e^{i k_{z} f(t)}\right|^{2} d t=1
$$

For $\omega_{0}=0$ one has $\lambda_{j}^{2}=k_{z}^{2}\left(\beta_{j}^{2}-1\right)$, where $\beta_{j}=v_{0} \sqrt{\varepsilon_{j}} / c, j=0,1$, and flux (22) is nonzero if the Cherenkov condition for the external medium is fulfilled, $\beta_{1}>1$. Passing to integration over $\omega=k_{z} \mathrm{v}_{0}$, we obtain

$$
I=\frac{4 q^{2} v_{0}}{\pi^{2} \rho_{1}^{2}} \int_{\beta_{1}^{2}>1} d \omega \frac{1}{\varepsilon_{1} \omega\left|W_{\varepsilon}\left(J_{0}, H_{0}\right)\right|^{2}}, \quad \omega_{0}=0 .
$$

It is easy to show that this expression coincides with the formula presented, for example, in Ref. [21] for the radiation of a charge moving parallel to the axis of a cylindrical channel in a dielectric. Introducing the angle $\vartheta$ of the wave vector with the cylinder axis, from the relation $\omega=k_{z} \mathrm{v}_{0}$ we obtain $\cos \vartheta=\beta_{1}^{-1}$, i.e., the radiation described by Eq. (24) propagates under 
the Cherenkov angle of the external medium. When $\varepsilon_{0}=\varepsilon_{1}$, from Eq. (24) we obtain the well-known formula for the intensity of the Cherenkov radiation in a homogeneous medium.

Now consider the general case, when $\omega_{0} \neq 0$. First we analyze the term with $n=0$ in formula (22). Analogously to deriving of Eq. (24), it is easy to show that the corresponding contribution to the radiation intensity is determined by the formula

$$
I_{n=0}=\frac{4 q^{2} v_{0}}{\pi^{2} \rho_{1}^{2}} \int_{\beta_{1}^{2}>1} d \omega \frac{\left|g_{0}\left(\omega / v_{0}\right)\right|^{2}}{\varepsilon_{1} \omega\left|W_{\varepsilon}\left(J_{0}, H_{0}\right)\right|^{2}} .
$$

This radiation propagates under the Cherenkov angle of the external medium and its intensity differs from the intensity of the uniformly moving charge, given by Eq. (24), by the presence of the frequency-dependent additional weight factor $\left|g_{0}\left(\omega / v_{0}\right)\right|^{2}$.

Now we proceed to the terms with $n \neq 0$ in formula (22). From the condition $\lambda_{1}^{2}>0$ we have the following quadratic inequality with respect to $k_{z}$ :

$$
k_{z}^{2}\left(1-\beta_{1}^{-2}\right)+2 k_{z} \frac{n \omega_{0}}{v_{0}}+\frac{n^{2} \omega_{0}^{2}}{v_{0}^{2}}>0 .
$$

Let the Cherenkov condition be not fulfilled initially for the drift velocity of the charge: $\beta_{1}<1$. In this case we obtain from this inequality:

$$
k_{z} \in\left(-\frac{n \omega_{0} \sqrt{\varepsilon_{1}}}{c\left(1+\beta_{1}\right)}, \frac{n \omega_{0} \sqrt{\varepsilon_{1}}}{c\left(1-\beta_{1}\right)}\right) .
$$

It is convenient to introduce a new variable $\vartheta$ according to

$$
k_{z}=\frac{n \omega_{0}}{c} \frac{\sqrt{\varepsilon_{1}} \cos \vartheta}{1-\beta_{1} \cos \vartheta}
$$

where from relation $(27)$ it follows that $\vartheta \in(0, \pi)$. Then from expression (10) we have

$$
\omega_{n}\left(k_{z}\right)=\frac{n \omega_{0}}{1-\beta_{1} \cos \vartheta}, n=1,2 \ldots
$$

Note that in accordance with Eqs. (28) and (29) the quantities $k_{z}$ and $\omega_{n}\left(k_{z}\right)$ are connected by the relation $k_{z}=\omega_{n}\left(k_{z}\right) \sqrt{\varepsilon_{1}} \cos \vartheta / c$.

Now consider the case $\beta_{1}>1$, when the solution of inequality (26) has the following form:

$$
k_{z} \in\left(-\infty,-\frac{n \omega_{0} \sqrt{\varepsilon_{1}}}{c\left(\beta_{1}-1\right)}\right) \cup\left(-\frac{n \omega_{0} \sqrt{\varepsilon_{1}}}{c\left(\beta_{1}+1\right)}, \infty\right) .
$$

Introducing again the variable $\vartheta$ according to expression (28), we ensure that $\vartheta \in\left(0, \vartheta_{0}\right) \cup\left(\vartheta_{0}, \pi\right)$, where $\vartheta_{0}=\arccos \left(\beta_{1}^{-1}\right)$ is the corresponding Cherenkov angle for the drift velocity. Relations between the variables $k_{z}, \omega_{n}\left(k_{z}\right)$, and $\vartheta$ now are the same as for $\beta_{1}<1$. At large distances from the charge trajectory the dependence of elementary waves on the space time coordinates has the form $\exp \left[\omega_{n}\left(k_{z}\right) \sqrt{\varepsilon_{1}}\left(\rho \sin \vartheta+z \cos \vartheta-c t / \sqrt{\varepsilon_{1}}\right) / c\right]$, which describes the wave with the frequency

$$
\omega_{n}=\left|\omega_{n}\left(k_{z}\right)\right|=\frac{n \omega_{0}}{\left|1-\beta_{1} \cos \vartheta\right|},
$$

propagating at the angle $\vartheta$ to the $z$-axis. Formula (10) describes the normal Doppler effect in the cases $\beta_{1}<1$ and $\beta_{1}>1, \vartheta>\vartheta_{0}$ and anomalous Doppler effect in the case $\beta_{1}>1, \vartheta<\vartheta_{0}$. By making use of the formulas given above, the expressions for the $\lambda_{j}$ can be written as

$$
\lambda_{0}=\frac{n \omega_{0}}{c} \frac{\sqrt{\varepsilon_{0}-\varepsilon_{1} \cos ^{2} \vartheta}}{1-\beta_{1} \cos \vartheta}, \quad \lambda_{1}=\frac{n \omega_{0}}{c} \frac{\sqrt{\varepsilon_{1}} \sin \vartheta}{1-\beta_{1} \cos \vartheta} .
$$


Taking into account these remarks and passing from integration over $k_{z}$ to integration over $\vartheta$, we obtain the following expression for the radiation intensity:

$$
I_{n \neq 0}=\sum_{n=1}^{\infty} \int \frac{d I_{n}}{d \Omega} d \Omega, \quad \frac{d I_{n}}{d \Omega}=\frac{2 q^{2} c}{\pi^{3} \rho_{1}^{2} \varepsilon_{1}^{3 / 2}} \frac{\left|g_{n}(n u)\right|^{2}}{\left|W_{\varepsilon}\left(J_{0}, H_{0}\right)\right|^{2}\left|1-\beta_{1} \cos \vartheta\right| \cos ^{2} \vartheta},
$$

where $d \Omega=\sin \vartheta d \vartheta d \phi$ is the element of solid angle and the notation

$$
u=\frac{\omega_{0}}{c} \frac{\sqrt{\varepsilon_{1}} \cos \vartheta}{1-\beta_{1} \cos \vartheta}
$$

is introduced. To obtain the radiation intensity at the angle $\vartheta=\pi / 2$, note that for $n \neq 0$ and $k_{z} \rightarrow 0$ from formula (11) one has

$$
g_{n}\left(k_{z}\right) \approx i k_{z} f_{n}, \quad f_{n}=\frac{1}{T} \int_{-T / 2}^{T / 2} f(t) e^{-i n \omega_{0} t} d t,
$$

with $f_{n}$ being the Fourier transform of the function $f(t)$. Now from Eqs. (33) and (34) one finds

$$
\frac{d I_{n}}{d \Omega}=\frac{2 q^{2} \omega_{0}^{2}}{\pi^{3} c \rho_{1}^{2} \sqrt{\varepsilon_{1}}} \frac{n^{2}\left|f_{n}\right|^{2}}{\left|W_{\varepsilon}\left(J_{0}, H_{0}\right)\right|^{2}}, \quad \lambda_{j}=\frac{n \omega_{0}}{c} \sqrt{\varepsilon_{j}}, \quad j=0,1 \quad \vartheta=\frac{\pi}{2} .
$$

By taking into account Eqs. (28), (29), and (32), from formula (17) one obtains the following relation between the electric and magnetic fields for the radiation at $n$th harmonic:

$$
\mathbf{E}_{n}=\frac{1}{\sqrt{\varepsilon_{1}}}\left[\mathbf{H}_{n} \mathbf{n}\right]
$$

where $\mathbf{n}=(\sin \vartheta, 0, \cos \vartheta)$ is the unit vector in the propagation direction. Hence, the radiation is linearly polarized and the polarization plane goes through the vectors $\mathbf{n}$ and $\mathbf{v}_{0}$.

The total intensity of the radiation for the longitudinal oscillator moving with a constant drift velocity along the axis of a dielectric cylinder can be written as the sum

$$
I=I_{n=0}+I_{n \neq 0}
$$

where the first term on the right is given by formula (25) and describes the radiation with a continuous spectrum propagating at the Cherenkov angle of the external medium, if the condition $\beta_{1}>1$ is fulfilled (for $\beta_{1}<1$ this term is absent). The second term describes the radiation, which for a given angle $\vartheta$ has a discrete spectrum determined by formula (31). With allowance for the dispersion of the dielectric permittivity, this term does not contribute to the radiation at the Cherenkov angle. This is connected with the fact that for a given $n>0$ the frequency defined by Eq. (31), tends to infinity as $\vartheta$ approaches $\vartheta_{0}$ and hence beginning from a certain frequency the Cherenkov condition ceases to be fulfilled. The angles for which the dispersion should be taken into account, are determined implicitly from the condition $\omega_{n} \geq \omega_{d}$ by using formula (31) and frequency dependence of the permittivity $\varepsilon_{1}=\varepsilon_{1}\left(\omega_{n}\right)$, where $\omega_{d}$ is the characteristic frequency of the dispersion.

Substituting $\varepsilon_{1}=\varepsilon_{0}$, from the formulas given above we obtain the corresponding expressions for an oscillator moving in a homogeneous medium. In particular, by taking into account the formula

$$
W_{\varepsilon}\left(J_{0}, H_{0}\right)=-\frac{2 i c\left(1-\beta_{1} \cos \vartheta\right)}{\pi \rho_{1} n \omega_{0} \sqrt{\varepsilon_{1}} \sin \vartheta}, \quad \varepsilon_{0}=\varepsilon_{1},
$$

from Eq. (33) for the corresponding radiation intensity one obtains

$$
I_{n \neq 0}^{(0)}=\sum_{n=1}^{\infty} \int \frac{d I_{n}}{d \Omega} d \Omega, \quad \frac{d I_{n}^{(0)}}{d \Omega}=\frac{q^{2} n^{2} \omega_{0}^{2}}{2 \pi c \sqrt{\varepsilon_{1}}} \frac{\tan ^{2} \vartheta\left|g_{n}(n u)\right|^{2}}{\left|1-\beta_{1} \cos \vartheta\right|^{3}} .
$$


Let us consider the radiation intensity given by Eq. (33) for large values of the harmonic $n$. The behaviour of the function $g_{n}(n u)$ for large $n$ can be estimated by using the stationary phase method. To do this we note that this function has the form

$$
g_{n}(n u)=\frac{1}{T} \int_{-T / 2}^{T / 2} e^{i n S(t)} d t, \quad S(t)=u f(t)-\omega_{0} t .
$$

The asymptotic behaviour of the integral for large values $n, n \rightarrow \infty$, is essentially different in dependence wether the function $S(t)$ has a stationary point, being the solution of the equation $S^{\prime}(t)=0$. By using expression (34) for $u$, this equation can be written in terms of the particle velocity $v(t)$ as

$$
\frac{v(t)}{c} \sqrt{\varepsilon_{1}} \cos \vartheta=1, \quad v(t)=v_{0}+f^{\prime}(t) .
$$

It follows from here that, if for the particle velocity $v(t)$ the Cherenkov condition is not fulfilled, the subintegrand in Eq. (41) has no stationary points and for $f(t) \in C^{\infty}$ the integral is exponentially suppressed for large values of the harmonic number $n$. When the Cherenkov condition is fulfilled the main term of the asymptotic expansion of integral in Eq. (41) is determined by the contribution of the stationary point and in accordance with the standard formula in the stationary phase method, one has $g_{n}(n u) \propto 1 / \sqrt{n}, n \rightarrow \infty$. In this case in the absence of the dispersion the radiation intensity linearly increases with increasing $n$ and in addition to the radiation from an oscillating charge one has also the Cherenkov radiation. To identify the angular regions where the Cherenkov radiation propagates, let us denote by $v_{+}$and $v_{-}$maximal and minimal values of the function $v(t)$ and

$$
\beta_{ \pm}=v_{ \pm} \sqrt{\varepsilon_{1}} / c
$$

The angular regions where the Cherenkov radiation propagates are as follows:

$$
\begin{aligned}
& \vartheta<\vartheta_{+}, \vartheta_{+} \equiv \arccos \frac{1}{\beta_{+}} \text {for }-1<\beta_{-}<1<\beta_{+} \\
& \vartheta_{-}<\vartheta<\vartheta_{+}, \vartheta_{-} \equiv \arccos \frac{1}{\beta_{-}} \text {for } \beta_{-}>1 \\
& \vartheta>\vartheta_{-}, \quad \text { for } \beta_{-}<-1, \quad \beta_{+}<1 \\
& \vartheta<\vartheta_{+} \cup \vartheta>\vartheta_{-} \text {for } \beta_{+}>1, \quad \beta_{-}<-1 .
\end{aligned}
$$

In the first two cases the Cherenkov radiation propagates in the inward direction to the $z$-axis. In the last two cases the Cherenkov radiation in the region $\vartheta>\vartheta_{-}$propagates in the backward direction. As we have mentioned, the radiation intensity in regions (44) linearly increases with increasing $n$. However, note that large values $n$ correspond to large frequencies for which the dispersion becomes important. Hence, for the radiation in regions (44) the value of the harmonic $n$ for which the radiation intensity has a maximum is determined by the dispersion through the conditions $\beta_{+}>1$ or $\left|\beta_{-}>1\right|$. Here the situation is similar to the case of the ordinary Cherenkov radiation.

By taking into account formula (40) for the radiation in a homogeneous medium with permittivity $\varepsilon_{1}$, expression (33) for the radiation intensity at $n$th harmonic in the presence of a dielectric cylinder may be written in the following form

$$
\frac{d I_{n}}{d \Omega}=\frac{d I_{n}^{(0)}}{d \Omega} F_{n}(\vartheta),
$$

where the inhomogeneity factor is given by the formula

$$
F_{n}(\vartheta)=\frac{4 c^{2}\left|W_{\varepsilon}\left(J_{0}, H_{0}\right)\right|^{-2}}{\pi^{2} \rho_{1}^{2} \varepsilon_{1} \omega_{n}^{2} \sin ^{2} \vartheta} .
$$


Consider the dependence of this factor on the cylinder radius in the limit $\left|\lambda_{j}\right| \rho_{1} \gg 1$, when the wavelength for the radiation is much less than the cylinder radius. Using the asymptotic expressions for the cylindrical functions for large values of the argument and taking into account formulas (32), for the inhomogeneity factor we get

$$
F_{n}(\vartheta)=\frac{2 \sqrt{\varepsilon_{0} \varepsilon_{1}-\cos ^{2} \vartheta}}{\sin \vartheta}\left[1+\frac{\varepsilon_{0}^{2} \sin ^{2} \vartheta}{\varepsilon_{1}^{2}\left(\varepsilon_{0} / \varepsilon_{1}-\cos ^{2} \vartheta\right)}+\left(1-\frac{\varepsilon_{0}^{2} \sin ^{2} \vartheta}{\varepsilon_{1}^{2}\left(\varepsilon_{0} / \varepsilon_{1}-\cos ^{2} \vartheta\right)}\right) \sin \left(2\left|\lambda_{0}\right| \rho_{1}\right)\right]^{-1}
$$

for $\cos ^{2} \vartheta<\varepsilon_{0} / \varepsilon_{1}\left(\lambda_{0}^{2}>0\right)$ and

$$
F_{n}(\vartheta)=\frac{4 \sqrt{\cos ^{2} \vartheta-\varepsilon_{0} \varepsilon_{1}}}{\sin \vartheta} e^{-2\left|\lambda_{0}\right| \rho_{1}}\left[1+\frac{\varepsilon_{0}^{2} \sin ^{2} \vartheta}{\varepsilon_{1}^{2}\left(\cos ^{2} \vartheta-\varepsilon_{0} / \varepsilon_{1}\right)}\right]^{-1}
$$

for $\cos ^{2} \vartheta>\varepsilon_{0} / \varepsilon_{1}\left(\lambda_{0}^{2}<0\right)$. As we see, in the second case the intensity exponentially decreases with increasing $\rho_{1}$. This is caused by the fact that for $\varepsilon_{0} / \varepsilon_{1}<1$ the angle arccos $\sqrt{\varepsilon_{0} / \varepsilon_{1}}$ corresponds to the angle of total internal reflection, and in the limit of the geometric optics the beams incident from inside on the cylinder surface cannot propagate at the angles $\cos ^{2} \vartheta<\varepsilon_{0} / \varepsilon_{1}$ in the external medium.

In the opposite limit, when the wavelength for the radiation is much larger than the cylinder radius $\left|\lambda_{j}\right| \rho_{1} \ll 1$, using the asymptotic expressions for cylindric functions, it is easy to show that from Eq. (33) one can immediately derive the radiation intensity in a homogeneous medium with the permittivity $\varepsilon_{1}$ (formula $(40)$ ), i.e., $F_{n}(\vartheta) \rightarrow 1$. For fixed values of $\varepsilon_{j}$ and $v_{0}$ the inhomogeneity factor $F_{n}$ is a function on $n \omega_{0} \rho_{1} / c$ and $\vartheta$ :

$$
F_{n}=F\left(\frac{n \omega_{0} \rho_{1}}{c}, \vartheta\right) .
$$

In Fig. 1 we have plotted this function for the charge drift velocity $v_{0}=0.9 \mathrm{c}$ in two different cases: $\varepsilon_{0}=3, \varepsilon_{1}=1$ (left figure) and $\varepsilon_{0}=1, \varepsilon_{1}=3$ (right figure). As we see, in the first case the presence of cylinder can essentially increase the radiation intensity. In the left graph it is clearly seen the exponential suppression of the inhomogeneity factor with increasing $n \omega_{0} \rho_{1} / c$ for angles $\vartheta<\arccos \sqrt{\varepsilon_{0} / \varepsilon_{1}} \approx 0.96$.

\section{Examples of the oscillatory motion}

In this section we will consider two examples of the oscillation law, corresponding to the harmonic oscillations in the laboratory and proper reference frames.

\subsection{Harmonic oscillation in the laboratory frame}

First of all let us consider the case when the charged particle oscillates as an harmonic oscillator in the laboratory frame with the amplitude $z_{0}$ :

$$
f(t)=z_{0} \sin \left(\omega_{0} t\right)
$$

From formula (11) it can be easily seen that for this type of motion one has

$$
g_{n}\left(k_{z}\right)=J_{n}\left(k_{z} z_{0}\right) .
$$

Hence, in this case the radiation intensity is given by formulas (25) and (33) with the function $g_{n}\left(k_{z}\right)$ given by Eq. (51). In particular, for $\vartheta=\pi / 2$ the radiation intensity is nonzero only at the fundamental harmonic $n=1$. Due to the well-known properties of the Bessel function 

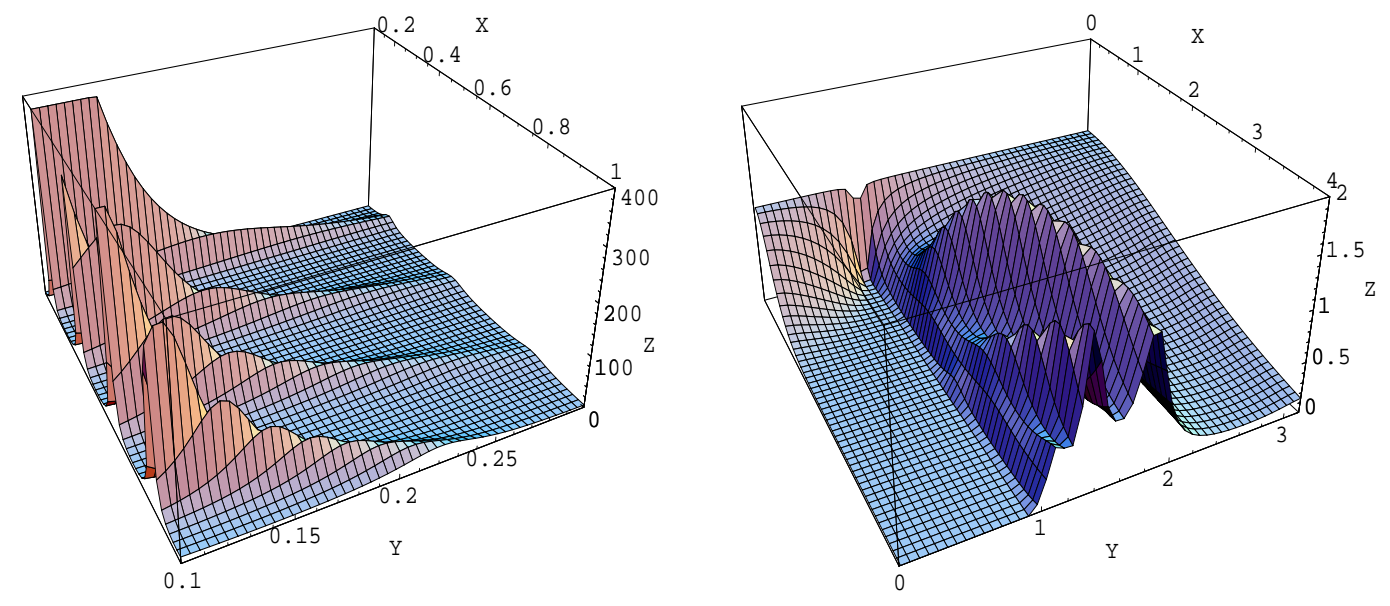

Figure 1: The inhomogeneity factor $Z=F_{n}$ as a function on $X=n \omega_{0} \rho_{1} / c$ and $Y=\vartheta$ for $v_{0}=0.9 \mathrm{c}$. Left figure corresponds to the values $\varepsilon_{0}=3, \varepsilon_{1}=1$ and right figure corresponds to $\varepsilon_{0}=1, \varepsilon_{1}=3$.

$J_{n}(n z)$, the behavior of the radiation intensity as a function of $n$ depends essentially on the sign of the quantity $1-\left(u z_{0}\right)^{2}$. By using formula (34), this quantity can be presented in the form

$$
1-w^{2}=\frac{1-\beta_{-} \cos \vartheta}{\left(1-\beta_{1} \cos \vartheta\right)^{2}}\left(1-\beta_{+} \cos \vartheta\right), \quad w=u z_{0},
$$

where we use notation (43) with

$$
v_{ \pm}=v_{0} \pm \omega_{0} z_{0}
$$

If the Cherenkov condition for the maximum velocity of the charge is not fulfilled, $\beta_{+}<1$, one has $w<1$. By using Debye's asymptotic expansion for the function $J_{n}(n w)$, it can be seen that for large values of the harmonic $n$ the radiation intensity behaves as

$$
I_{n} \sim \frac{n}{x}\left(\sqrt{\frac{1-x}{1+x}} e^{x}\right)^{2 n}, \quad x=\sqrt{1-w^{2}} .
$$

It follows from here that for $x \simeq 1$ the spectral distribution has maximum at

$$
n \simeq n_{\max }=\left(1-w^{2}\right)^{-3 / 2},
$$

and is exponentially suppressed for larger harmonics.

For $\beta_{+}>1$ regions for values of the angle $\vartheta$ exist for which $w>1$. In these regions in addition to the radiation from an oscillating charge, one has also the Cherenkov radiation. The angular regions where the Cherenkov radiation propagates are determined by formulas (44). As follows from the formula for the radiation intensity in combination with Eq. (51), for a given harmonic $n$ the corresponding intensity vanishes at angles determined from the equation $\left|u z_{0}\right|=j_{n, l} / n, l=1,2, \ldots$, where $j_{n, l}$ are the positive zeros of the Bessel function: $J_{n}\left(j_{n, l}\right)=0$. If the Cherenkov condition in the external medium for the maximum velocity of the charge $v_{0}+\omega_{0} z_{0}$ is not fulfilled, then $\left|u z_{0}\right|<1$, and as follows from the relation $j_{n, l}>n$ for the zeros of the Bessel function, this equation has no solutions. If the Cherenkov condition is fulfilled, then the radiation intensity at a given harmonic $n$ vanishes at the angles $\vartheta$ determined from the formula

$$
\cos \vartheta_{n, l}=\frac{c}{\sqrt{\varepsilon_{1}}\left(v_{0} \pm n \omega_{0} z_{0} / j_{n, l}\right)}
$$


These angles do not depend on the dielectric permittivity of the cylinder and, hence, are the same for the radiation in the presence of the cylinder and in a homogeneous medium.

Consider now formula (40) for the radiation intensity in a homogeneous medium with the function $g_{n}\left(k_{z}\right)$ from (51). First we analyze the case when the Cherenkov condition is not fulfilled for the maximal velocity of the charge, $\beta_{+}<1$, when $\left|u z_{0}\right|<1$. If we neglect the dispersion, then in Eq. (40) the series over $n$ is summarized by means of the standard formula [22]

$$
\sum_{n=1}^{\infty} n^{2} J_{n}^{2}(n w)=\frac{w^{2}\left(w^{2}+4\right)}{16\left(1-w^{2}\right)^{7 / 2}}, \quad w<1 .
$$

Then from Eq. (40) for the angular distribution of the radiation intensity we obtain

$$
\frac{d I^{(0)}}{d \vartheta}=\frac{q^{2} \omega_{0}^{2}}{16 c \sqrt{\varepsilon_{1}}} \frac{\tan ^{2} \vartheta \sin \vartheta}{\left|1-\beta_{1} \cos \vartheta\right|^{3}} \frac{w^{2}\left(w^{2}+4\right)}{\left(1-w^{2}\right)^{7 / 2}} .
$$

For $\varepsilon_{1}=1$ this formula can be obtained from the general formula for the radiation of a fourdimensional harmonic oscillator presented, for instance, in Refs. [3, 4]. For $\beta_{+}>1$, a range of values of $\vartheta$ exists, where $\left|u z_{0}\right|>1$ (see Eqs. (44)), and in the absence of dispersion the series over $n$ in Eq. (40) diverges. Thus, in this case, in order to obtain a finite result for the radiation intensity, the consideration of the dispersion is necessary. In the limit of low velocities of the oscillatory motion $\left(\omega_{0} z_{0} \ll c\right)$ the radiation intensity for a given harmonic at angles different from the Cherenkov one tends to zero as $\left(\omega_{0} z_{0} / c\right)^{2 n}$ for both homogeneous and inhomogeneous cases.
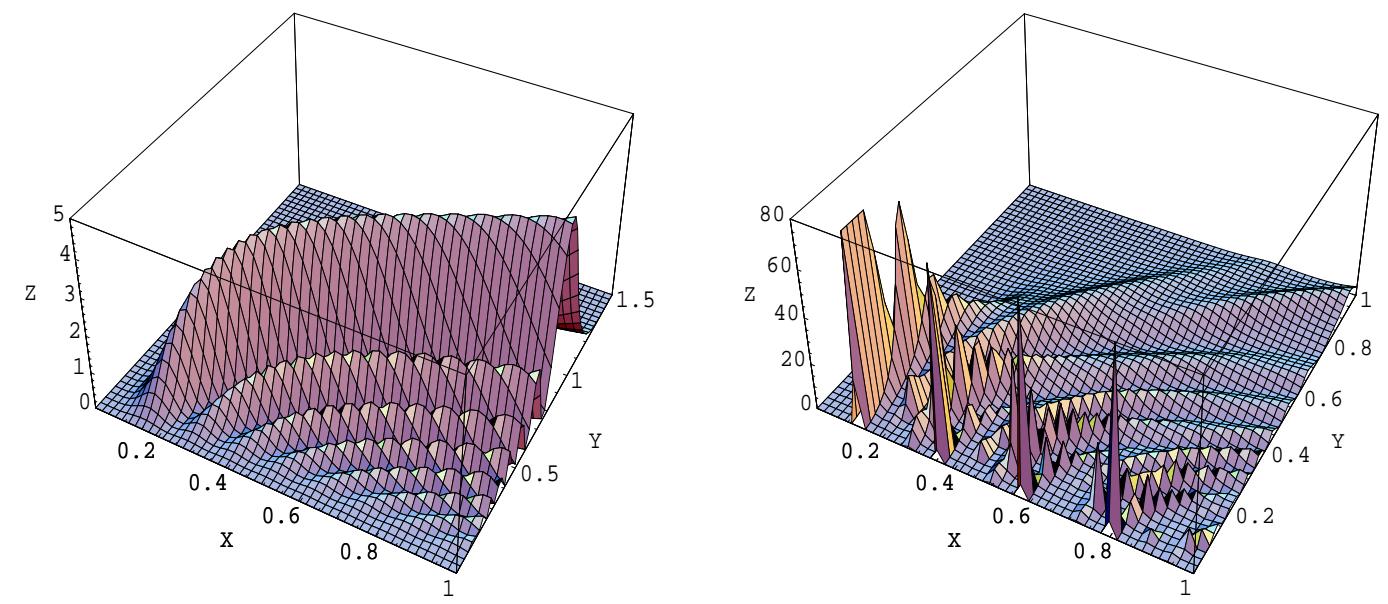

Figure 2: Number of emitted quanta per solid angle, $Z=\left(\hbar c \sqrt{\varepsilon_{1}} / q^{2}\right)\left(2 \pi / \omega_{0}\right) d N_{n} / d \Omega$, as a function on $X=\omega_{0} z_{0} \sqrt{\varepsilon_{1}} / c$ and $Y=\vartheta$ for $v_{0} \sqrt{\varepsilon_{1}} / c=0.9, n=3$ in a homogeneous medium, $\varepsilon_{0}=\varepsilon_{1}$ (left figure) and in presence of a cylinder with $\varepsilon_{0} / \varepsilon_{1}=3, \rho_{1}=z_{0}$ (right figure).

We have performed numerical calculation for the angular distribution of the density of number of quanta

$$
\frac{d N_{n}}{d \Omega}=\frac{1}{\hbar \omega_{n}} \frac{d I_{n}}{d \Omega}
$$

for several values of the harmonic number $n$. Figures 2 and 3 show the results of these calculations for the oscillator radiation in a homogeneous medium and in presence of a cylinder with dielectric permittivity $\varepsilon_{0} / \varepsilon_{1}=3$. As is seen from these figures the presence of the cylinder can essentially increase the radiation intensity. 


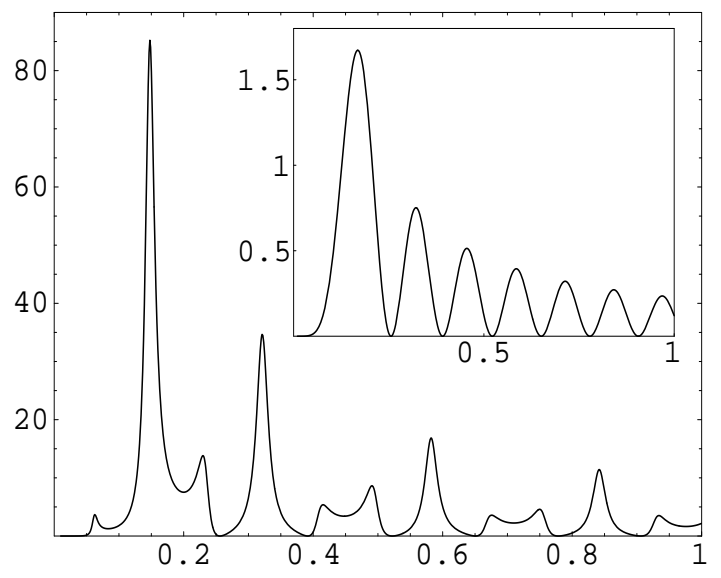

Figure 3: The same quantity as in Fig. 2 as a function on $\omega_{0} z_{0} \sqrt{\varepsilon_{1}} / c$ for $\vartheta=0.2, v_{0} \sqrt{\varepsilon_{1}} / c=0.9$, $n=3, \varepsilon_{0} / \varepsilon_{1}=3, \rho_{1}=z_{0}$. The graph in the right-up corner corresponds to the same quantity for the oscillator radiation in a homogeneous medium $\left(\varepsilon_{0}=\varepsilon_{1}\right)$.

\subsection{Harmonic oscillation in the proper reference frame}

In this subsection we consider the radiation from a charge that executes harmonic oscillations in the reference frame which moves at the constant velocity $v_{0}$. In the comoving coordinate system, the coordinates of the particle are given by

$$
z^{\prime}=a^{\prime} \sin \left(\omega_{0}^{\prime} t^{\prime}\right), \quad x^{\prime}=y^{\prime}=0 .
$$

The radiation generated by this kind of oscillator in the vacuum (the case $\varepsilon_{0}=\varepsilon_{1}=1$ ) has been studied in Ref. [17] (see also Ref. [4]). In the laboratory reference frame the motion of the oscillator is described by formula (1), where the function $f(t)$ is defined in the parametric form as

$$
f(t)=a^{\prime} \sqrt{1-\beta^{2}} \sin \left(\omega_{0}^{\prime} t^{\prime}\right), \quad t=\frac{1}{\sqrt{1-\beta^{2}}}\left(t^{\prime}+\frac{a^{\prime} v_{0}}{c^{2}} \sin \left(\omega_{0}^{\prime} t^{\prime}\right)\right) \quad \beta=\frac{v_{0}}{c} .
$$

This function is periodic with the period $T=2 \pi / \omega_{0}$, where $\omega_{0}$ is related to the proper frequency $\omega_{0}^{\prime}$ by the standard formula $\omega_{0}=\omega_{0}^{\prime} \sqrt{1-\beta^{2}}$. For the function defined by Eq. (61) one has $f(-t)=-f(t)$, and the function $g_{n}\left(k_{z}\right)$ is real. Substituting into Eq. (11) expressions (61) and performing to the new integration variable $x=\omega_{0}^{\prime} t^{\prime}$, one finds

$$
g_{n}\left(k_{z}\right)=\frac{1}{\pi} \int_{0}^{\pi}(1+A \cos x) \cos (n B \sin x-n x) d x,
$$

where

$$
A=\frac{a^{\prime} \omega_{0}^{\prime} v_{0}}{c^{2}}, \quad B=\frac{k_{z} a^{\prime}}{n} \sqrt{1-\beta^{2}}-A
$$

Note that using formula (28), the expression for the coefficient $B$ can be presented in the form

$$
B=\frac{\omega_{0}^{\prime} a^{\prime}}{c \sqrt{\varepsilon_{1}}} \frac{\varepsilon_{1} \cos \vartheta-\beta_{1}}{1-\beta_{1} \cos \vartheta} .
$$

The integral in Eq. (62) can be evaluated by using the standard formula from Ref. [22]:

$$
g_{n}\left(k_{z}\right)=J_{n}(n B) \frac{\left(1-\beta^{2}\right) \varepsilon_{1} \cos \vartheta}{\varepsilon_{1} \cos \vartheta-\beta_{1}} .
$$


Substituting this into general formula (33), for the angular distribution of the radiation intensity at $n$th harmonic one finds

$$
\frac{d I_{n}}{d \Omega}=\frac{2 q^{2} c \sqrt{\varepsilon_{1}}}{\pi^{3} \rho_{1}^{2}} \frac{\left(1-\beta^{2}\right)^{2} J_{n}^{2}(n B)}{\left|W_{\varepsilon}\left(J_{0}, H_{0}\right)\right|^{2}\left|1-\beta_{1} \cos \vartheta\right|\left(\varepsilon_{1} \cos \vartheta-\beta_{1}\right)^{2}} .
$$

By taking into account formula (39), for the radiation intensity of an oscillator moving in a homogeneous medium with dielectric permittivity $\varepsilon_{1}$, one obtains

$$
\frac{d I_{n}^{(0)}}{d \Omega}=\frac{q^{2} \varepsilon_{1}^{3 / 2}}{2 \pi c} \frac{n^{2} \omega_{0}^{2}\left(1-\beta^{2}\right)^{2} \sin ^{2} \vartheta J_{n}^{2}(n B)}{\left|1-\beta_{1} \cos \vartheta\right|^{3}\left(\varepsilon_{1} \cos \vartheta-\beta_{1}\right)^{2}} .
$$

For $\varepsilon_{1}=1$ this expression coincides with the formula derived in Ref. [17].

As in the previous example, the behavior of the radiation intensity (66) is essentially different for the cases $B<1$ and $B>1$. Note that from the expression for $B$ it follows that

$$
1-B^{2}=\frac{1-\left(v_{0} \omega_{0}^{\prime} a^{\prime} / c^{2}\right)^{2}}{\left(1-\beta_{1} \cos \vartheta\right)^{2}}\left(1-\beta_{-} \cos \vartheta\right)\left(1-\beta_{+} \cos \vartheta\right)
$$

where $\beta_{ \pm}$are defined by Eq. (43) with

$$
v_{ \pm}=\frac{v_{0} \pm \omega_{0}^{\prime} a^{\prime}}{1 \pm v_{0} \omega_{0}^{\prime} a^{\prime} / c^{2}}
$$

If for the maximal velocity of the charge, $v_{+}$, the Cherenkov condition is not satisfied, $\beta_{+}<1$, from (68) one has $B<1$ and the radiation intensity behaves as in Eq. (54) with the replacement $w \rightarrow B$. In this case in the corresponding formula for the radiation in a homogeneous medium, given by Eq. (67), the series over $n$ can be summarized with the help of formula (57) assuming that the dispersion is absent:

$$
\frac{d I^{(0)}}{d \Omega}=\sum_{n=1}^{\infty} \frac{d I_{n}^{(0)}}{d \Omega}=\frac{q^{2} \omega_{0}^{2} \sqrt{\varepsilon_{1}}}{32 \pi c}\left(\frac{\omega_{0}^{\prime} a^{\prime}}{c}\right)^{2} \frac{\left(1-\beta^{2}\right)^{2} \sin ^{2} \vartheta}{\left|1-\beta_{1} \cos \vartheta\right|^{5}} \frac{\left(4+B^{2}\right)}{\left(1-B^{2}\right)^{7 / 2}},
$$

where $1-B^{2}$ is given by Eq. (68). For $\beta_{+}>1$ regions for $\vartheta$ exist with $B>1$. In these regions in addition to the radiation from an oscillating charge one has also the Cherenkov radiation. These regions are determined by relations (44). In Fig. 4 we have plotted the number of emitted quanta (59) as a function on $\omega_{0}^{\prime} a^{\prime} / c$ and $\vartheta$ for $v_{0}=0.9 c, n=3$ in vacuum (left figure) and in presence of a cylinder with dielectric permittivity $\varepsilon_{0}=3$ (right figure). In Fig. 5 the number of radiated quanta is presented as a function on $\omega_{0}^{\prime} a^{\prime} / c$ for $\vartheta=0.2$. The values for the other parameters are the same as in Fig. 4. As we see from the presented examples the presence of the dielectric cylinder can lead to the essential enhancement of the radiation intensity.

\section{Conclusion}

In the present paper we have considered the influence of a dielectric cylinder on the electromagnetic radiation from a longitudinal oscillator with uniform drift along the axis of the cylinder. By using the Green function, for the general case of the oscillation law we have derived formulas for the electromagnetic fields, Eqs. (15), (16), and for the angular-frequency distribution of the radiation intensity. The latter is a sum of two terms (see Eq. (38)). The first one, given by formula (25), is present when the Cherenkov condition for dielectric permittivity of the exterior medium and drift velocity is satisfied, $\beta_{1}>1$, and describes the radiation propagating under the Cherenkov angle of the external medium. The corresponding intensity differs from the radiation 

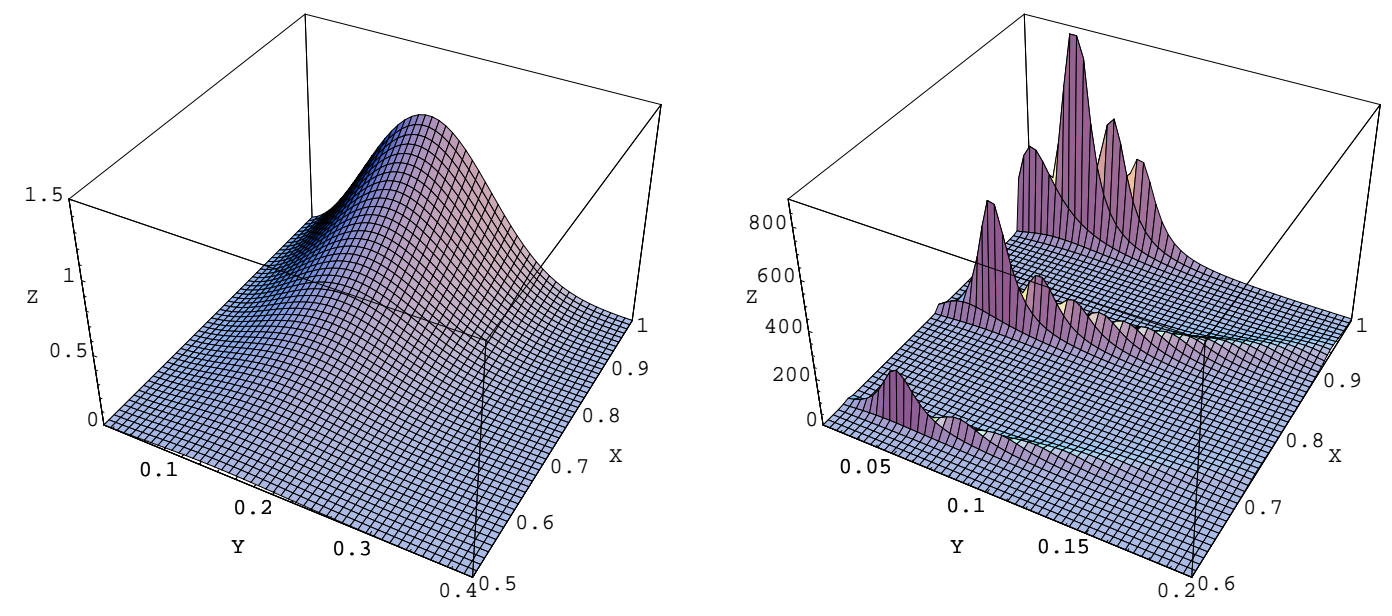

Figure 4: Number of emitted quanta per solid angle, $Z=\left(\hbar c / q^{2}\right)\left(2 \pi / \omega_{0}\right) d N_{n} / d \Omega$, as a function on $X=\omega_{0}^{\prime} a^{\prime} / c$ and $Y=\vartheta$ for $v_{0}=0.9 c, n=3$ in vacuum $\varepsilon_{0}=\varepsilon_{1}=1$ (left figure) and in presence of a cylinder with $\varepsilon_{0}=3, \rho_{1}=a^{\prime}$ (right figure).

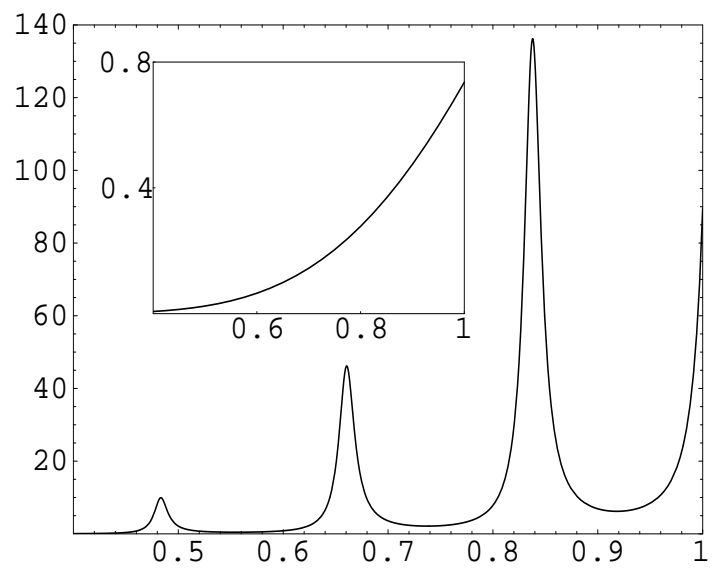

Figure 5: The same quantity as in Fig. 4 as a function on $\omega_{0}^{\prime} a^{\prime} / c$ for $\vartheta=0.2, v_{0}=0.9 c, n=3$, $\varepsilon_{0}=3, \rho_{1}=a^{\prime}$. The graph in the left-up corner corresponds to the same quantity for the oscillator radiation in vacuum $\left(\varepsilon_{0}=\varepsilon_{1}=1\right)$. 
intensity of a charge uniformly moving along the axis of a dielectric cylinder by presence of the frequency-dependent factor $\left|g_{0}\left(\omega / v_{0}\right)\right|^{2}$, where the function $g_{n}(x)$ is determined by the oscillation law and is defined by formula (11). The second term in the total radiation intensity (38) is given by formula (33) and describes the radiation, which for a given propagation direction has a discrete spectrum determined by formula (31). If the Cherenkov conditions for the maximal and minimal velocities of the charge and dielectric permittivity of the exterior medium are not satisfied, $\left|\beta_{ \pm}\right|<1$, the Cherenkov radiation is absent and for large values of the harmonic $n$ the radiation intensity is exponentially suppressed. The corresponding behaviour is essentially different when $\beta_{+}>1$ or $\left|\beta_{-}\right|>1$. In these cases there are angular regions, defined by relations (44), there in addition to the radiation from an oscillating charge one has also the Cherenkov radiation. In these regions, for large values of the harmonic number the radiation intensity linearly increases with increasing $n$. However, large values $n$ correspond to large frequencies for which the dispersion becomes important. The values of harmonic $n$ with the maximal radiated intensity are determined by the specific dispersion law for the dielectric permittivity of the exterior medium. In presence of a cylinder the radiation intensity at a given harmonic differs from the corresponding intensity in a homogeneous medium by the inhomogeneity factor $F_{n}(\vartheta)$, defined by formula (46). Our calculations for specific values of the parameters have shown that this factor can essentially exceed the unity. An example is presented in Fig. 1. This provides a possibility for the essential enhancement of the radiation intensity caused by the presence of a dielectric cylinder. In Sec. 4 we have specified the general formula of the radiation intensity for two examples of the oscillation function. The first one, described in subsection 4.1, corresponds to a charged particle oscillating as a harmonic oscillator in the laboratory reference frame. The second example, considered in subsection 4.2, corresponds to a charge that executes harmonic oscillations in the reference frame which moves at a constant velocity. The corresponding functions $g_{n}\left(k_{z}\right)$ are determined by formulas (51) and (62). Our numerical calculations for specific examples have shown that in both these cases the presence of a dielectric cylinder can essentially increase the radiation intensity to compared with the radiation in a homogeneous medium. Note that the results obtained in this paper are valid also in the case when the oscillator moves inside

a hole along the axis of a dielectric cylinder assuming that the radius of the hole is much less than the radiated wavelength.

\section{Acknowledgement}

The authors are grateful to Professor A. R. Mkrtchyan for general encouragement and to Professor L. Sh. Grigoryan, S. R. Arzumanyan, H. F. Khachatryan for many stimulating discussions. A. A. S. acknowledges the hospitality of the Department of Theoretical Electrotechnics of the Berlin Technical University and Professor Heino Henke for helpful discussions and useful comments. The work has been supported by Grant No. 1361 from Ministry of Education and Science of the Republic of Armenia.

\section{References}

[1] P. Luchini and H. Motz, Undulators and Free-electron Lasers (Clarendon, 1990).

[2] Ch. A. Brau, Free-electron Lasers (Academic Press, Boston, 1990).

[3] M. M. Nikitin and V. Ya. Epp, Undulator Radiation (Energoatomizdat, Moscow, 1988, in Russian). 
[4] V. Ya. Epp, in Synchrotron Radiation Theory and Its Developments edited by V. A. Bordovitsyn (World Scientific, Singapore, 1999).

[5] A. R. Mkrtchyan, L. Sh. Grigoryan, A. N. Didenko, A. A. Saharian, and A. G. Mkrtchyan, Izv. Akad. Nauk Arm. SSR Fiz. 24, 62 (1989)[Sov. J. Contemp. Phys. 24, 10 (1989)]; A. R. Mkrtchyan, L. Sh. Grigoryan, A. N. Didenko, and A. A. Saharian, Sov. Phys. JTP 61, 21 (1991); A. R. Mkrtchyan, L. Sh. Grigoryan, A. A. Saharian, and A. N. Didenko, Acustica 75, 1984 (1991).

[6] A. A. Saharian, A. R. Mkrtchyan, L. A. Gevorgian, L. Sh. Grigoryan, and B. V. Khachatryan, Nucl. Instrum. Methods B 173, 211 (2001).

[7] L. Sh. Grigoryan, A. S. Kotanjyan, and A. A. Saharian, Izv. Akad. Nauk Arm. SSR Fiz. 30, 239 (1995) [Sov. J. Contemp. Phys. 30, 1 (1995)].

[8] S. R. Arzumanian, L. Sh. Grigoryan, Kh. V. Kotanjyan, and A. A. Saharian, Izv. Akad. Nauk Arm. SSR Fiz. 30, 106 (1995) [Sov. J. Contemp. Phys. 30, 12 (1995)].

[9] L. Sh. Grigoryan, H. F. Khachatryan , and S. R. Arzumanyan, Izv. Akad. Nauk Arm. SSR Fiz. 33, 267 (1998) [Sov. J. Contemp. Phys. 33, 1 (1998)], cond-mat/0001322.

[10] A. S. Kotanjyan, H. F. Khachatryan , A . V. Petrosyan, and A. A. Saharian, Izv. Akad. Nauk Arm. SSR Fiz. 35, (2000) [Sov. J. Contemp. Phys. 35, 1 (2000)].

[11] A. S. Kotanjyan and A. A. Saharian, Izv. Akad. Nauk Arm. SSR Fiz. 36, (2001) [Sov. J. Contemp. Phys. 36, 7 (2001)].

[12] A. S. Kotanjyan and A. A. Saharian, Mod. Phys. Lett. A 17, 1323 (2002).

[13] A. S. Kotanjyan, Nucl. Instrum. Methods B 201, 3 (2003).

[14] B. V. Khachatryan, Izv. Vissh. Uchebn. Zaved, Radiofiz. 6, 904 (1963).

[15] F. A. Kostanyan and O. S. Mergelyan, Izv. Akad. Nauk Arm. SSR Fiz. 6, 481 (1971).

[16] F. A. Kostanyan and O. S. Mergelyan, Izv. Akad. Nauk Arm. SSR Fiz. 6, 472 (1971); 12, 179 (1977).

[17] A. B. Kukanov and E. K. Suleiman, Izv. Vissh. Uchebn. Zaved, Radiofiz. 11, 7 (1975).

[18] K. A. Barsukov, E. A. Begloyan, E. M. Laziev, and H. V. Ryazantseva, The radiation from a charged particle, making oscillations along the direction of motion through the waveguide (Yerevan Physics Institute, Preprint EFI-964(14)-87 ).

[19] A. A. Saharian and A. S. Kotanjyan, Izv. Akad. Nauk Arm. SSR Fiz. 38, 288 (2003).

[20] Handbook of Mathematical Functions, edited by M. A. Abramovitz and I. A. Stegun (Dover, New York, 1972).

[21] B. M. Bolotovsky, Usp. Fiz. Nauk 75, 295 (1961).

[22] A. P. Prudnikov, Yu. A. Brychkov, and O. I. Marichev, Integrals and Series, Vol. 2: Special Functions (Harwood Academic, Newark, NJ, 1986). 